\title{
Correlation of SWAP (short wavelength automated perimetry) with OCT (optical coherence tomography in pre perimetric glaucoma
}

\author{
Punit Singh $^{1}$, Anshi Rathod ${ }^{2, *}$, Zeel Patel ${ }^{3}$, Nidhi Vithlani ${ }^{4}$, Bhoomi Thakkar ${ }^{5}$ \\ ${ }^{\mathbf{1}}$ Associate Professor, ${ }^{2} 3^{\text {rd }}$ Year Resident, ${ }^{3,4,5} 2^{\text {nd }}$ Year Resident, Dept. of Ophthalmology, Dhiraj Hospital, Sumandeep \\ Vidyapeeth, Vadodara, Gujarat, India \\ *Corresponding Author: Anshi Rathod \\ Email: anshirathod@gmail.com
}

\begin{abstract}
Purpose: To find out correlation of swap and OCT in preperimetric glaucoma patient coming to Dhiraj general hospital. Materials and Methods: A complete ophthalmological examination will be done including uncorrected and best corrected visual acuity, slit lamp examination, central corneal thickness measurement by pachymetry, applanation tonometry, gonioscopy and dilated fundus examination and Humphrey perimetry followed by SWAP and FDT.

Result: In the present study a total of 50 patients were screened. Out of this 22 patients had a CDR of 0.5 with healthy neuroretinal rim (HNRR) in both the eyes. One patient had 0.5 CDR with inferior thinning in both the eyes. 8 patients had a CDR of 0.6 with HNRR and 1 had 0.6 CDR with inferior thinning. 2 patients had a large cup to disc ratio of 0.7 CDR but the neuro retinal rim on clinical examination appeared to be healthy. 14 patients had a large cup to disc ratio of 0.7 in both the eyes. Out of this 8 had inferior thinning of the neuro-retinal rim and 6 had superior thinning of the neuro retinal rim. 2 patients included in this study had $0.8 \mathrm{CDR}$ with one having superior thinning and one having inferior thinning of the neuro retinal rim. The accuracy of OCT in detecting even the smallest nerve fiber layer defect was much higher than seen on SWAP. About 52\% showed normal OCT as compared to perimetry group which was $60 \%$ and about $48 \%$ of patients showed affected nerve fiber layer thinning as compared to perimetry where visual field changes were $40 \%$.

Conclusion: In this study it was concluded that SD-OCT is better in detection very early stages of glaucoma where SAP or SWAP modality of perimetry does not detect.

The sensitivity of OCT is much higher than perimetry and can help in detecting the suspects of glaucoma. Patients at with high risk (family history, smokers, large cup to disc ratio etc) should be screen for both OCT and SWAP. This will help us for manage patients better as they will be detected at a very early stage.
\end{abstract}

Keywords: Pre perimetric glaucoma, SD-OCT, SWAP.

\section{Introduction}

Glaucoma is a noteworthy irreversible reason for visual deficiency, representing $1 / 6^{\text {th }}$ portion of all reasons for irreversible blindness. ${ }^{1}$ It is a dynamic optic neuropathy in which visual weakness happens because of retinal ganglion cells (RGC) death. ${ }^{2}$

Open-Angle Glaucoma (OAG) is a conceivably blinding visual condition that is portrayed by a dynamic optic neuropathy with trademark visual field loss with open points on gonioscopy. ${ }^{3}$ It is additionally connected with raised intraocular pressure. ${ }^{4}$

Edge conclusion glaucoma (ACG) results from decrease in watery outflow. ${ }^{3}$ This is because of appositional or synechial conclusion of the front chamber point. This prompts amassing of watery inside the foremost chamber and IOP is hence raised. ${ }^{3}$ This will in the long run prompt optic nerve harm and visual field loss. Edge conclusion can present as intense assault or ceaseless in nature. Intense edge conclusion emergency is a crisis and need earnest treatment. ${ }^{4}$

Ordinary Tension glaucoma is type a typical sort in which there is ordinary intra visual weight. ${ }^{5}$ It is ceaseless and nature and dynamic which quietly prompts optic neuropathy, retinal nerve fiber layer diminishing and visual field surrenders. ${ }^{5}$

Standard robotized perimetry otherwise called white boost against a white foundation (W-W perimetry). ${ }^{7}$ It recognizes a visual field deformity when around $40 \%$ of the retinal ganglion cells (RGCs) have been lost. Standard mechanized perimetry is ideally used to recognize harm at prior phases of ganglion cell loss. It gives us data of irreversible nature of vision loss in glaucoma. ${ }^{8}$

SWAP otherwise called blue-on-yellow perimetry (B-Y). ${ }^{9}$ It distinguishes early visual field loss before changes seen on standard white-on-white (W-W) perimetry. Around 5\% to $10 \%$ retinal ganglion cells associate with the kiniocelluar pathway of the horizontal geniculate body (LGB). ${ }^{9}$ These cells are lost is early glaucoma. Standard robotized perimetry does not test these subpopulation of retinal ganglion cells. These cells are delicate to blue light and in this manner on SWAP they are disengaged and tried. ${ }^{10}$

SWAP utilizes a thin band blue-light upgrade and yellow-foundation brightening to underline the reaction attributes of the blue- yellow pathway. ${ }^{7}$ This utilitarian test has been appeared to recognize visual field irregularities in patients at high danger of creating glaucoma and in patients with glaucomatous optic neuritis when standard (white-on-white) visual fields are still inside ordinary points of confinement. ${ }^{10}$ Often times, harm revealed by SWAP goes before standard field loss by at least three years. ${ }^{10}$ SWAP likewise uncovers a more noteworthy spatial degree of visual 
field harm in glaucoma patients than standard perimetry. ${ }^{11}$

Be that as it may, on standard mechanized perimetryto analyze glaucoma at the optic circle or on the visual field, ganglion cell loss needs to happen. Up to half of neighborhood ganglion cell focus might be lost to glaucoma before visual field variations from the norm are recognized utilizing customary standard mechanized perimetry (SAP). ${ }^{12}$

SD-OCT is quickly developing with quicker examining velocities, 3D picture obtaining designs, reproducible enrollment and propelled division calculations. The clinical utility of SD-OCT in glaucoma primarily centers around the accompanying three parameters. ${ }^{13}$

1. Retinal nerve fiber layer,

2. Optic nerve head,

3. Ganglion cell complex.

The numeric qualities for all parameters are shading coded as white, green, yellow, or red, with the yellow and red speaking to, $<5 \%$ and $<1 \%$, individually contrasted with the regularizing database..$^{13}$ However, the inconstancy of the encompassing structures and the nearness of existing together pathology may affect dependable estimation. Accordingly, the upgraded execution of SD-OCT takes into account the appraisal of macular parameters for glaucoma assessment on the grounds that the macula has the most noteworthy centralization of RGC in the retina (roughly half of the RGC of here.) ${ }^{14}$ Furthermore, given the capacity of SD-OCT to deliver 3D datasets, there is currently potential to survey $\mathrm{ONH}$ parameters for glaucoma assessment with more prominent exactness and enhanced movement location in back to back testing by exact picture enrollment. There are right now a few financially accessible SD-OCT gadgets with fluctuating parameters and one of a kind highlights. ${ }^{10}$

SD-OCT measures and evaluates the RNFL thickness. It does by ascertaining the locale between the inward constraining film (ILM) and RNFL fringe. 3.4 $\mathrm{mm}$ distance across hover of RNFL information is extricated to make what is alluded to as the TSNIT outline, (predominant, nasal, substandard, temporal). ${ }^{12}$ The optic nerve is in the focal point of this region. It is known as the cirrus RNFL map. ${ }^{12}$ It is An output information which speaks to a $6 \times 6 \mathrm{~mm}$ 3D shape. The focal point of the 3D square is the optic plate. The guide shows the thickness of retinal nerve fiber esteems by quadrants and clock hours. The predominant and sub-par nerve fiber packs gives a pinnacle appearance on the map. ${ }^{13}$

SD-OCT naturally ascertains the optic plate region and neuro retinal edge. It does as such by denoting the frameworks of the optic nerve head, the optic glass, and plate edges. It for the most part considers the vertical glass to-circle proportions. This isn't conceivable with time domain-OCT. ${ }^{15}$
The ganglion cell layer estimation additionally assumes a vital job in movement and location of the illness. The ganglion cell layer is thickest in the peri macular region. ${ }^{14}$ It has been seen in numerous glaucomatous eye that there is a reduction in the aggregate macular thickness. This is because of diminishing of the ganglionic cell layer. In the Cirrus delineate the measure through its Ganglion Cell Analysis (GCA) which comprises of the ganglion cell layer (GCL) and inward plexiform layer (IPL) ${ }^{16}$

It has been demonstrated by different investigations that noteworthy loss of nerve fiber layer happens much before the adjustments in the visual field loss. ${ }^{17}$ SD-OCT assumes an each vital job in preperimetric glaucoma as SD-OCT can get the adjustments in the nerve fiber layer exceptionally early. When the loss of nerve fiber layer compares to the trademark visual field loss it affirms the conclusion of glaucoma. ${ }^{17}$

\section{Aim and Objective}

To assess effectiveness of optical coherence tomography (OCT) and short wave automated perimetry (SWAP) in diagnosing pre perimetric glaucoma.

\section{Materials and Methods}

The study was conducted in the department of ophthalmology at Dhiraj Hospital, Sumandeep Vidyapeeth, Vadodara.

Study Design: Total number of cases will be 50 cases of preperimetric glaucoma.

1. Study will be clinical, prospective and comparative.

2. Basic method of data collection will be serial and observational.

\section{Inclusion Criteria}

1. Age group more than 35 year both male and female

2. Family history of glaucoma

3. Myopes and hypermetropes both

4. Patient having suspicious disc changes ( 0.5 cup to disc ratio and more) on clinical examination

5. Patient having glaucomatous disc changes ( 0.5 cup to disc ratio and more) on clinical examination

6. Willing to fill the informed consent form.

\section{Exclusion Criteria}

1. Chronic ocular surface disease

2. Ocular media opacities. (Significant cataract)

3. History of any ocular surgery (Lasik, AGS)

4. Extended contact lens use

5. Occupation involving exposure to extreme hot climate, radiation, smoke etc

6. Patient taking drugs like Antihistamines, antidepressants, oral contraceptive pill.

7. Pregnant women.

8. Not willing to fill informed consent shit. 
Methods: A complete history of the patient was taken who report to Dhiraj hospital - ophthalmology OPD. History of any medications, systemic disorders, ocular surgeries, family history of glaucoma, etc will thoroughly asked.

A complete ophthalmological examination was done. It included: unaided visual acuity, best corrected visual acuity for near and far, slit lamp examination, undilated and dilated fundus examination with $90 \mathrm{D}$ and 20D respectively, colour vision by Ishihara chart, contrast sensitivity by pellirobsonchart, central corneal thickness measurement by ultrasonic pachymetry, Goldmann applanation tonometry to measure the intraocular pressure, gonioscopy to evaluate the angles of anterior chamber, Humphrey visual fields - Short wave automated perimetry (SWAP) and SD- OCT 3D disc.

\section{Result}

In the present study a total of 50 patients were screened. These patients were satisfying as inclusion and exclusion criteria of the study. Out of this 22 patients had a CDR of 0.5 with healthy neuro-retinal rim (HNRR) in both the eyes. One patient had 0.5 CDR with inferior thinning in both the eyes. 8 patients had a CDR of 0.6 with HNRR and 1 had 0.6 CDR with inferior thinning. 2 patients had a large cup to disc ratio of $0.7 \mathrm{CDR}$ but the neuro retinal rim on clinical examination appeared to be healthy. 14 patients had a large cup to disc ratio of 0.7 in both the eyes. Out of this 8 had inferior thinning of the neuro-retinal rim and 6 had superior thinning of the neuro retinal rim. 2 patients included in this study had $0.8 \mathrm{CDR}$ with one having superior thinning and one having inferior thinning of the neuro retinal rim.

Goldmann applanation tonometry was carried out in both the eyes of all 50 patients. The mean deviation of the intraocular pressure was about 24.16 in the right eye and 24.34 in the left eye.

Ultrasonic pachymetry was also carried out in both the eyes of all patients to know the central corneal thickness. The mean pachymetry of both the eyes was about 516. Pachymetry plays a very important role in progression and risk of developing glaucoma.

Humphrey Visual Fields SWAP was carried in all the patients who were selected. It was done undilated pupil with best corrected visual acuity. In this study about $60 \%$ of patients with different cup to disc ratio showed normal perimetry (SWAP) and about $40 \%$ had shown visual field changes on perimetry.

SD- OCT was done using 3D optic disc modality. The accuracy of OCT in detecting even the smallest nerve fiber layer defect was much higher than seen on SWAP. About 52\% showed normal OCT as compared to perimetry group which was $60 \%$ and about $48 \%$ of patients showed affected nerve fiber layer thinning as compered to perimetry where visual field changes were $40 \%$.
The following tables represents the result of the study.

Table 1

\begin{tabular}{|l|c|c|}
\hline $\begin{array}{c}\text { Applanation } \\
\text { Tonometer }\end{array}$ & Mean & SD \\
\hline OD & 24.16 & 7.44 \\
\hline OS & 24.34 & 7.98 \\
\hline
\end{tabular}

Table 2

\begin{tabular}{|l|c|c|}
\hline Pachymetry & Mean & SD \\
\hline OD & 516.06 & 16.35 \\
\hline OS & 516.02 & 14.73 \\
\hline
\end{tabular}

Table 3

\begin{tabular}{|l|c|c|}
\hline \multicolumn{1}{|c|}{ Gender } & Mean Age & SD \\
\hline Male & 36.84 & 5.78 \\
\hline Female & 33.08 & 7.74 \\
\hline Total & 35.94 & 6.42 \\
\hline
\end{tabular}

Table 4

\begin{tabular}{|c|c|c|c|}
\hline \multicolumn{2}{|c|}{ OCT } & Mean & SD \\
\hline \multirow{4}{*}{ OD } & Superior & 114.64 & 16.39 \\
\cline { 2 - 4 } & Temporal & 65.36 & 8.35 \\
\cline { 2 - 4 } & Inferior & 122.46 & 17.80 \\
\cline { 2 - 4 } & Nasal & 70.42 & 12.26 \\
\cline { 2 - 4 } & Average & 93.31 & 12.09 \\
\hline \multirow{4}{*}{ OS } & Superior & 113.59 & 16.31 \\
\cline { 2 - 4 } & Temporal & 65.70 & 12.29 \\
\cline { 2 - 4 } & Inferior & 118.38 & 14.26 \\
\cline { 2 - 4 } & Nasal & 71.24 & 12.10 \\
\cline { 2 - 4 } & Average & 92.25 & 11.80 \\
\hline
\end{tabular}

Table 5

\begin{tabular}{|l|c|c|}
\hline \multicolumn{1}{|c|}{ Fundus Examination } & $\mathbf{N}$ & $\mathbf{\%}$ \\
\hline 0.5 CDR HNRR & 22 & $44.00 \%$ \\
\hline 0.5 CDR INF THINNING & 1 & $2.00 \%$ \\
\hline 0.6 CDR HNRR & 8 & $16.00 \%$ \\
\hline 0.6 CDR INF THINNING & 1 & $2.00 \%$ \\
\hline 0.7 CDR HNRR & 2 & $4.00 \%$ \\
\hline 0.7 CDR INF THINNING & 8 & $16.00 \%$ \\
\hline 0.7 CDR SUP THINNING & 6 & $12.00 \%$ \\
\hline 0.8 CDR INF THINNING & 1 & $2.00 \%$ \\
\hline 0.8 CDR SUP THINNING & 1 & $2.00 \%$ \\
\hline Total & 50 & $100.00 \%$ \\
\hline
\end{tabular}


Table 6

\begin{tabular}{|l|c|c|c|c|c|c|c|c|c|}
\hline Fundus Examination & $\mathbf{N}$ & \multicolumn{5}{|c|}{ Perimetry } & \multicolumn{4}{|c|}{ OCT } \\
\cline { 2 - 11 } & & Normal & $\mathbf{\%}$ & Positive & $\mathbf{\%}$ & Normal & $\mathbf{\%}$ & Positive & $\%$ \\
\hline 0.5 CDR HNRR & 22 & 22 & $100.00 \%$ & 0 & $0.00 \%$ & 20 & $90.91 \%$ & 2 & $9.09 \%$ \\
\hline $\begin{array}{l}0.5 \text { CDR INF } \\
\text { THINNING }\end{array}$ & 1 & 0 & $0.00 \%$ & 1 & $100.00 \%$ & 0 & $0.00 \%$ & 1 & $100.00 \%$ \\
\hline 0.6 CDR HNRR & 8 & 7 & $87.50 \%$ & 1 & $12.50 \%$ & 6 & $75.00 \%$ & 2 & $25.00 \%$ \\
\hline $\begin{array}{l}0.6 \text { CDR INF } \\
\text { THINNING }\end{array}$ & 1 & 0 & $0.00 \%$ & 1 & $100.00 \%$ & 0 & $0.00 \%$ & 1 & $100.00 \%$ \\
\hline 0.7 CDR HNRR & 2 & 1 & $50.00 \%$ & 1 & $50.00 \%$ & 0 & $0.00 \%$ & 2 & $100.00 \%$ \\
\hline $\begin{array}{l}0.7 \text { CDR INF } \\
\text { THINNING }\end{array}$ & 8 & 0 & $0.00 \%$ & 8 & $100.00 \%$ & 0 & $0.00 \%$ & 8 & $100.00 \%$ \\
\hline $\begin{array}{l}0.7 \text { CDR SUP } \\
\text { THINNING }\end{array}$ & 6 & 0 & $0.00 \%$ & 6 & $100.00 \%$ & 0 & $0.00 \%$ & 6 & $100.00 \%$ \\
\hline $\begin{array}{l}0.8 \text { CDR INF } \\
\text { THINNING }\end{array}$ & 1 & 0 & $0.00 \%$ & 1 & $100.00 \%$ & 0 & $0.00 \%$ & 1 & $100.00 \%$ \\
\hline $\begin{array}{l}0.8 \text { CDR SUP } \\
\text { THINNING }\end{array}$ & 1 & 0 & $0.00 \%$ & 1 & $100.00 \%$ & 0 & $0.00 \%$ & 1 & $100.00 \%$ \\
\hline \multicolumn{1}{|c|}{ Total } & 50 & 30.00 & $60.00 \%$ & 20.00 & $40.00 \%$ & 26.00 & $52.00 \%$ & 24.00 & $48.00 \%$ \\
\hline
\end{tabular}

The mean difference between the sensitivity of OCT and perimetry was about $8 \%$. Thus concluding the higher sensitivity of OCT in pre perimetric glaucoma. The retinal nerve fibers in the inferior quadrant was the easiest parameter affected.

Males showed more changes than the female group in this study. And the changes were also seen more in the elderly age group as compared to younger patients.

\section{Discussion}

Glaucoma is a neurodegenerative disease caused by progressive retinal ganglion cell (RGC) loss associated with characteristic structural changes in the optic nerve and retinal nerve fiber layer (RNFL). ${ }^{16}$ These changes are irreversible and chronic. The neural insult can result in functional losses and decrease in vision-related quality of life. ${ }^{17}$ Detection of progression and estimation of rates of disease deterioration are essential in order to evaluate risk of functional impairment and establish treatment strategies. ${ }^{17}$

As the harm from glaucoma is irreversible, it is vital that the illness is recognized at a beginning period, before huge field loss has grown, with the goal that the danger of visual hindrance and related horribleness can be minimized. ${ }^{19}$

The finding of glaucoma relies upon acknowledgment of trademark auxiliary changes to the optic nerve head $(\mathrm{ONH})$ and retinal nerve-fiber layer (RNFL). ${ }^{19}$ Psychophysical tests, the best quality level of which is standard mechanized perimetry (SAP), ought to likewise be utilized to identify irregularities of visual capacity demonstrative of glaucoma. ${ }^{19}$

In the beginning times of glaucoma, large-diameter ganglion cells might be lost specifically, ${ }^{19,20}$ despite the fact that this idea remains controversial. ${ }^{20}$ Furthermore, a ganglion cell populace, which has moderately few numbers, will have little save when loss happens.
Ganglion cell axons anticipating from blue-on cells inside the koniocellular pathway are around half bigger than those in red-or green-touchy pathways and are less in number. ${ }^{21}$ Tests that objective them (eg, short wavelength robotized perimetry [SWAP]) can accordingly recognize glaucomatous harm quite a long while sooner than SAP. ${ }^{22}$

SWAP focuses on the short-wavelength- delicate cones and pathway. At the ganglion cell level, the patient's reaction to this test is undoubtedly intervened by the little bi-stratified blue- yellow ganglion cells that involve around $9 \%$ of the aggregate populace of retinal ganglion cells. ${ }^{23}$ The test gives a dynamic scope of around $35 \mathrm{~dB}$ and $15 \mathrm{~dB}$ of disengagement before the following most delicate system can recognize the objective, undoubtedly the center wavelength- touchy pathway cells. ${ }^{25}$ SWAP utilizes a pale blue (440-nm wavelength) tight band focus of $1.8^{\circ}$ displayed for 200 $\mathrm{ms}$ on a brilliant $(100 \mathrm{~cd} / \mathrm{m} 2)$ yellow foundation. ${ }^{26}$

The demonstrative capacities of SD-OCT for segregating among solid and glaucomatous eyes utilizing normal RNFL thickness have been accounted for to have a territory under recipient working attributes bend estimation of around 0.9. ${ }^{27}$ However, the separation capacity is reliant on the seriousness phase of glaucoma, with better execution in segregating among solid and more development sickness contrasted and separation of beginning periods of glaucoma. ${ }^{27}$

In this investigation a sum of 50 patients were screened. These patients were fulfilling as consideration and avoidance criteria of the examination. Out of this 22 patients had a CDR of 0.5 with healthy neuro-retinal edge (HNRR) in both the eyes. 1 quiet had 0.5 CDR with inferior thinning in both the eyes. 8 patients had a CDR of 0.6 with HNRR and 1 had 0.6 CDR with second rate diminishing. 2 patients had an extensive container to plate proportion of $0.7 \mathrm{CDR}$ however the 
neuro retinal edge on clinical examination seemed, by all accounts, to be sound. 14 patients had an expansive container to circle proportion of 0.7 in both the eyes. Out of this 8 had inferior diminishing of the neuroretinal edge and 6 had predominant diminishing of the neuro retinal edge. 2 patients incorporated into this examination had $0.8 \mathrm{CDR}$ with one having predominant diminishing and one having second rate diminishing of the neuro retinal edge.

Procurement of 3D pictures of the $\mathrm{ONH}$ district empowers exact and reproducible estimations of $\mathrm{ONH}$ parameters that include: plate and edge territory, glass to circle proportion, container volume and others. ${ }^{28} \mathrm{An}$ analytic ability consider with SD-OCT of glaucoma and age-coordinated solid controls announced that these ONH parameters can separate among sound and glaucomatous eyes like RNFL thickness. ${ }^{28}$ Another contemplate with glaucoma, preperimetric glaucoma and solid subjects showed that RNFL thickness was superior to anything any tried $\mathrm{ONH}$ parameter. ${ }^{28}$ The conflicting aftereffects of these two investigations might be credited to contrast in glaucoma seriousness inside the examination tests. Notwithstanding, the two investigations detailed comparable symptomatic capacity with edge territory and normal RNFL thickness in cutting edge glaucoma. The job of SDOCT ONH examination in glaucoma analysis is yet to be resolved. ${ }^{36}$

SD-OCT analytic investigations have exhibited that glaucomatous harm brings about diminishing of RNFL and GCIPL and additionally ONH basic changes that take into consideration separation among glaucoma and solid eyes. ${ }^{29}$ However, in a large portion of these investigations, the symptomatic exactness may not decipher when utilized in clinical practice for beginning period glaucoma recognition in light of the fact that the segregation examines are typically founded on separating sound eyes from eyes with set up glaucomatous visual field (VF) loss. ${ }^{30}$ An ongoing SDOCT ponder looked at the symptomatic capacity of RNFL, ONH and macular parameters for diagnosing preperimetric glaucoma in an observational partner with 13 years of development. ${ }^{30}$ The agents showed that RNFL parameters performed fundamentally superior to $\mathrm{ONH}$ and macular parameters for identifying preperimetric glaucomatous harm. ${ }^{31}$ It is conceivable that a mix of parameters from the different filtered areas can enhance demonstrative execution; be that as it may, this still can't seem to be assessed. ${ }^{36}$

Accordingly in our examination we found a distinction of $8 \%$ in the adequacy of identifying pre perimetric glaucoma between SWAP Humphrey perimetry and SD-OCT. Around $8 \%$ of patients who were clinically ordinary yet with a glass to plate proportion of more than 0.5 had indicated nerve fiber layer imperfection on OCT. Anyway their SWAP perimetry was typical demonstrating no visual field defects. The viability of OCT in diagnosing glaucoma in a beginning period assumes an imperative job. In examination among SAP and SWAP, it was discovered that SWAP methodology successfully indicated changes on perimetry in early glaucoma than Standard Automated Perimetry.

\section{Conclusion}

In this investigation it was reasoned that SD-OCT is better in recognition beginning times of glaucoma where SAP or SWAP methodology of perimetry does not identify.

The affectability of OCT is substantially higher than perimetry and can help in distinguishing the suspects of glaucoma. Patients at with high hazard (family history, smokers, expansive glass to circle proportion and so forth) ought to be screen for both OCT and SWAP. This will help us is early discovery and ending the movement of glaucoma and oversee them as ahead of schedule as could reasonably be expected.

\section{References}

1. Sihota Ramanjit, Tandon Radhika., editors. Parsons' Diseases of the Eye. 21st ed. Elsevier; 2011. p. 541.

2. Clement C, Goldberg I, Healey P, Graham S. Humphrey matrix frequency doubling perimetry for detection of visual-field defects in open-angle glaucoma. The British Journal of Ophthalmology. 2009;93(5):582-8.

3. Wolfs RCW Borger PHRamrattan RS. Changing views in open-angle glaucoma: definitions and prevalence: the Rotterdam Study. Invest Ophthalmol Vis Sci. 2000;41:3309- 3321.

4. Quigley HA, Enger C, Katz J, Sommer A, Scott R, Gilbert D. Risk factors for the development of Glaucomatous visual field loss in ocular hypertension. Archives of Ophthalmology. 1994;112(5):644-9.

5. Quigley HA, Addicks EM, Green RW. Optic nerve damage in human glaucoma. Archives of Ophthalmology. 1982;100(1):135-46.

6. Johnson C. The Glenn A Fry Award Lecture: early losses of visual function in glaucoma. Optom Vis Sci. 1995;7(2):359-70.

7. Sample P, Weinreb R. Color perimetry for assessment of primary open-angle glaucoma. Investigative ophthalmology \& visual science. 1990;31(9):1869-75.

8. Demirel S, Johnson C. Short wavelength automated perimetry (SWAP) in ophthalmic practice. Journal of the American Optometric Association. 1996;67(8):451-6.

9. Tatham AJ, Weinreb RN, Medeiros FA. Strategies for improving early detection of glaucoma: The combined structure-function index. Clinical Ophthalmology. 2014;8:611-21.

10. Quigley HA, Sanchez RM, Dunkelberger GR, L'Hernault NL, Baginski TA. Chronic glaucoma selectively damages large optic nerve fibers. Invest Ophthalmol Vis Sci. 1987;28:913-920.

11. Quigley HA, Dunkelberger GR, Green RW. Chronic human glaucoma causing selectively greater loss of large optic nerve fibers - Ophthalmology. Ophthalmology. 1988;95(3):357-63.

12. Yücel YH, Zhang Q, Gupta N, Kaufman PL, Weinreb RN. Loss of Neurons in Magnocellular and Parvocellular layers of the lateral Geniculate nucleus in glaucoma. Archives of Ophthalmology. 2000;118(3):378-84. 
13. Martin PR, White AJR, Goodchild AK, Wilder HD, Sefton AE. Evidence that blue-on cells are part of the third geniculocortical pathway in primates. Eur $J$ Neurosci. 1997;91536-1541.

14. de Monasterio FM Asymmetry of on- and off-pathways of blue-sensitive cones of the retina of macaques. Brain Res. 1979;16639-48.

15. Sample P, Johnson C, Haegerstrom-Portnoy G, Adams A. Optimum parameters for short-wavelength automated perimetry. Journal of Glaucoma. 1996;5(6):375-83.

16. Aref AA, Budenz DL. Spectral domain optical coherence tomography in the diagnosis and management of glaucoma. Ophthalmic Surg Lasers Imaging. 2010;41:S15-27.

17. Grewal DS, Tanna AP. Diagnosis of glaucoma and detection of glaucoma progression using spectral domain optical coherence tomography. Curr Opin Ophthalmol. 2013;24:150-161.

18. Leite MT, Rao HL, Weinreb RN. Agreement among spectral-domain optical coherence tomography instruments for assessing retinal nerve fiber layer thickness. Am J Ophthalmlol. 2011;151:85-92.

19. Chang RT, Knight OJ, Feuer WJ, Budenz DL. Sensitivity and specificity of time-domain vs spectral-domain optical coherence tomography in diagnosing early to moderate glaucoma. Ophthalmology. 2009;116:1257-1263.

20. Mwanza JC, Oakley JD, Budenz DL, Anderson DR. Ability of Cirrus HD-OCT Optic Nerve Head Parameters to Discriminate Normal from Glaucomatous Eyes. Ophthalmology. 2011;118:241-248.

21. Mwanza JC, Durbin MK, Budenz DL. Glaucoma diagnostic accuracy of ganglion cell-inner plexiform layer thickness: comparison with nerve fiber layer and optic nerve head. Ophthalmology. 2012;119:1151-1158.

22. Quigley HA, Dunkelberger GR, Green WR. Retinal ganglion cell atrophy correlated with automated perimetry in human eyes with glaucoma. Am J Ophthalmol. 1989;107(5):453-64.

23. Mwanza JC, Chang RT, Budenz DL. Reproducibility of peripapillary retinal nerve fiber layer thickness and optic nerve head parameters measured with cirrus HD-OCT in glaucomatous eyes. Invest Ophthalmol Vis Sci. 2010;51:5724-5730.

24. Leung CK, Yu M, Weinreb RN. Retinal nerve fiber layer imaging with spectral-domain optical coherence tomography: patterns of retinal nerve fiber layer progression. Ophthalmology. 2012;119:1858-1866.

25. Hood D, Kardon R. A framework for comparing structural and functional measurements of glaucomatous damage. Prog Retin Eye Res. 2007;26:688-710.

26. Chong GT, Lee RK. Glaucoma versus red disease: imaging and glaucoma diagnosis. Curr Opin Ophthalmol. 2012;23:79-88.
27. Leung CK, Yu M, Weinreb RN. Retinal nerve fiber layer imaging with spectral-domain optical coherence tomography: interpreting the RNFL maps in healthy myopic eyes. Invest Ophthalmol Vis Sci. 2012;53:71947200.

28. Kaliner E, Cohen MJ, Miron H. Retinal nerve fiber layer split bundles are true anatomic variants. Ophthalmology. 2007;114:2259-2264.

29. Leung CK, Yu M, Weinreb RN. Retinal nerve fiber layer imaging with spectral-domain optical coherence tomography: a prospective analysis of age-related loss. Ophthalmology. 2012;119:731-737.

30. Balasubramanian M, Bowd C, Vizzeri G. Effect of image quality on tissue thickness measurements with spectraldomain optical coherence tomography. Opt Express. 2009;17:4019-4036.

31. Mwanza JC, Bhorade AM, Sekhon N, et al. Effect of cataract and its removal on signal strength and peripapillary retinal nerve fiber layer optical coherence tomography measurements. J Glaucoma. 2011;20:37-43.

32. Langenegger SJ, Funk J, Toteberg-Harms M. Reproducibilty of retinal nerve fiber layer thickness measurements using the eye tracker and the retest function of Spectralis SD-OCT in glaucomatous and healthy control eyes. Invest Ophthalmol Vis Sci. 2011;52:3338-3344.

33. Ho J, Sull AC, Vuong LN. Assessment of artifacts and reproducibility across spectral- and time-domain optical coherence tomography devices. Ophthalmology. 2009;116:1960-1970.

34. Lee DW, Kim JM, Park KH. Effect of media opacity on retinal nerve fiber layer thickness measurements by optical coherence tomography. J Ophthalmol Vis Res. 2010;5:151-157.

35. Savini G, Barboni P, Parisis V. The influence of axial length on retinal nerve fibre layer thickness and optic-disc size measurements by spectral-domain OCT. $\mathrm{Br} J$ Ophthalmol. 2012;96:57-61.

36. Sharma N, Sony P, Gupta A. Effect of laser in situ keratomileusis and laser-assisted subepithelial keratectomy on retinal nerve fiber layer thickness. $J$ Cataract Refract Surg. 2006;32:446-450.

How to cite this article: Singh $\mathrm{P}$, Rathod A, Patel Z, Vithlani N, Thakkar B. Correlation of SWAP (short wavelength automated perimetry) with OCT (optical coherence tomography in pre perimetric glaucoma. Indian $\mathbf{J}$ Clin Exp Ophthalmol. 2018;4(4):535-540. 